\title{
Evaluating NAT2PRED for inferring the individual acetylation status from unphased genotype data Audrey Sabbagh*1,2, Pierre Darlu ${ }^{3,4}$ and Michel Vidaud ${ }^{1,2}$
}

Address: ${ }^{1}$ INSERM UMR745, Faculty of Pharmacy, University of Paris Descartes, Paris, France, ${ }^{2}$ Department of Biochemistry and Molecular Genetics, Beaujon hospital, AP-HP, Clichy, France, ${ }^{3}$ INSERM U535, Paul Brousse Hospital, Villejuif, France and ${ }^{4}$ University of Paris-Sud, UMRS535, Villejuif, France

Email: Audrey Sabbagh* - audrey.sabbagh@parisdescartes.fr; Pierre Darlu - pierre.darlu@inserm.fr; Michel Vidaud - michel.vidaud@parisdescartes.fr

* Corresponding author

Published: 3 I December 2009

BMC Medical Genetics 2009, 10:148 doi:10.1186/1471-2350-10-148
Received: II August 2009

Accepted: 31 December 2009

This article is available from: http://www.biomedcentral.com/I47/-2350/I0//48

(c) 2009 Sabbagh et al; licensee BioMed Central Ltd.

This is an Open Access article distributed under the terms of the Creative Commons Attribution License (http://creativecommons.org/licenses/by/2.0), which permits unrestricted use, distribution, and reproduction in any medium, provided the original work is properly cited.

\begin{abstract}
Background: Genetically determined differences in $\mathrm{N}$-acetylation capacity have proved to be important determinants of both the effectiveness of therapeutic response and the development of adverse drug reactions and toxicity during drug treatment. NAT2PRED is a web-server that allows a fast determination of NAT2 acetylation phenotype from genotype data without taking the extra step of reconstructing haplotypes for each individual (publicly available at http:// nat2pred.rit.albany.edu). However, the classification accuracy of NAT2PRED needs to be assessed before its application can be advocated at a large scale.

Methods: The ability of NAT2PRED to classify individuals according to their acetylation status (slow, intermediate and rapid acetylators) was evaluated in a worldwide dataset composed of 56 population samples (8,489 individuals) from four continental regions.

Results: NAT2PRED correctly identified slow acetylators with a sensitivity above $99 \%$ for all populations outside sub-Saharan Africa. Nevertheless, NAT2PRED showed a poor ability to distinguish between intermediate and rapid acetylators, with a classification error rate reaching up to $10 \%$ in the non-African samples.

Conclusion: NAT2PRED is an excellent tool to infer the individual acetylation status from NAT2 genotype data when the main interest is to distinguish slow acetylators from the others. This should facilitate the determination of the individual acetylation status in routine clinical practice and lead to better monitoring of risks associated with cancer and adverse drug reactions.
\end{abstract}

\section{Background}

The human acetylation polymorphism is one of the most intensively studied pharmacogenetic traits that underlie interindividual and interethnic differences in response to xenobiotics. Genetically determined differences in $\mathrm{N}$ acetylation capacity have proved to be important determinants of both the effectiveness of therapeutic response and the development of adverse drug reactions and toxicity during drug treatment $[1,2]$. Some of the drugs excreted by acetylation are crucial in the treatment of diseases representing a worldwide concern, such as tuberculosis, AIDS-related complex diseases, and hypertension. Moreover, numerous association studies have linked the acetylation phenotype to susceptibility to a variety of 
complex human diseases, the most consistent findings being those regarding urinary bladder cancer, asthma and other allergic disorders [3-6].

Single nucleotide polymorphisms (SNPs) in the coding region of the NAT2 gene determine the acetylation phenotype. Thanks to the well-established genotype-phenotype correlation at this locus, the individual acetylation status can be reliably predicted from the haplotype combination at NAT2, according to the acknowledged classification of NAT2 haplotypes into either low-activity or fully functional alleles (see the consensus NAT2 gene nomenclature website: http://www.louisville.edu/medschool/pharma cology/NAT.html). Slow, intermediate and rapid acetylators are defined as carriers of zero, one or two functional haplotypes, respectively. Many studies did not distinguish between rapid and intermediate acetylators, categorizing both types of subjects as rapid acetylators.

Determining the linkage phase of SNP alleles along the coding sequence of NAT2 is crucial to unequivocally assign an individual's multi-site NAT2 genotype to a particular combination of two multilocus haplotypes and to correctly infer his acetylation status. Ambiguous NAT2 genotyping data that may lead to patient misclassification appear indeed to be common in human populations [7]. Special analytical techniques have been designed to unambiguously determine the allocation of SNP alleles to either DNA strand. However, molecular haplotyping methods are labour-intensive and expensive and are not suitable for routine clinical applications. A cheap and straightforward alternative for haplotype reconstruction is the use of computational algorithms. Several studies have demonstrated the high effectiveness of haplotype reconstruction algorithms in the particular case of the NAT2 gene by comparing the computationally inferred haplotypes to the real ones resolved through the use of molecular haplotyping techniques [7-9]. But such in silico approaches may not be convenient to clinicians not familiar with computational haplotype analysis. Moreover, algorithmic techniques are statistical and require the analysis of a population rather than a single or a few individuals.

To circumvent these limitations, Kuznetsov et al. [10] have developed a web-server NAT2PRED that allows a fast determination of NAT2 acetylation phenotypes (slow, intermediate, and rapid) from the unphased genotype data at six polymorphic positions in NAT2. These six SNPs are the most commonly reported ones in surveys of NAT2 sequence variation in human populations [11]. NAT2PRED alleviates the need of reconstructing haplotypes by implementing a supervised pattern recognition method that was trained on the NAT2 genotyping data of 1,377 subjects of known acetylation status. This tool is publicly available http://nat2pred.rit.albany.edu and has a simple intuitive user interface. It showed a nearly perfect classification accuracy of $99.9 \%$ in a sample mostly composed of Caucasians [10]. However, it remains unclear to what extent this tool can be applied to individuals from any ethnicity since it was developed on a dataset where $94 \%$ of subjects were Caucasian. The accuracy of NAT2PRED needs to be assessed before its application can be advocated at a large scale.

The objective of the present study was to empirically evaluate the performance of NAT2PRED in a wide collection of population samples worldwide. To this end, we performed an extensive survey of the literature to identify those samples that were adequately genotyped for all the common SNPs in NAT2. In total the collected data consisted of 8,489 individuals from 56 human populations representing major geographic regions: sub-Saharan Africa (12 samples), Europe and North Africa (23), Central/South Asia (5), East Asia (13), and America (3). In each sample, NAT2 haplotypes were reconstructed using either molecular or computational methods. Therefore, these data provided an opportunity to compare, for each subject, the acetylation status inferred by NAT2PRED from the unphased NAT2 genotype data with the "true" one predicted from the pair of haplotypes reconstructed through molecular or computational haplotyping. NAT2PRED correctly identified slow acetylators with a sensitivity above $99 \%$ for all populations outside subSaharan Africa.

\section{Methods}

We selected from published reports up to November 2009 all the population samples that were genotyped for the seven most common SNPs in NAT2 (191G>A ( $r s 1801279), 282 \mathrm{C}>\mathrm{T}$ ( $\mathrm{rs} 1041983, \mathrm{rs} 59855457), 341 \mathrm{~T}>\mathrm{C}$ (rs1801280, rs56935242), 481C >T (rs1799929, rs60310310), 590G $>A$ (rs1799930, rs60190029), $803 \mathrm{~A}>\mathrm{G}$ (rs1208, rs56599719, rs58999469), and 857G>A (rs1799931, rs58803786)) and for which genotype frequency data were available in the paper. We also included those non-African samples that were genotyped for all SNPs except SNP 191G>A as this is rare in non-African populations. The final dataset used for the present study describe 56 population samples from throughout the world, representing 8,489 individuals. A summary description of the data set is provided in Additional File 1.

In each sample, NAT2 haplotypes were either directly resolved using molecular-haplotyping techniques (through allele-specific PCR and restriction mapping) or were computationally inferred from the unphased multilocus genotypes using statistical algorithms (based either on a parsimony, maximum-likelihood, or Bayesian approach). For some samples, a combination of the two 
approaches was used. The acetylation phenotype of each individual was then predicted by assuming that the homozygous or compound heterozygous genotype for two haplotypes of the series NAT2 *4, NAT2 * $11, N A T 2 * 12$ or $N A T 2 * 13$ results in the rapid acetylator status, the occurrence of one of these haplotypes in combination with a haplotype of the series NAT2 $* 5, N A T 2 * 6, N A T 2 * 7$ or $N A T 2 * 14$ results in the intermediate acetylator status and the occurrence of two haplotypes of the series NAT2 $* 5, N A T 2 * 6, N A T 2 * 7$ or NAT2 ${ }^{*} 14$ results in the slow acetylator phenotype.

Besides, NAT2PRED was applied to the unphased genotype data of each sample (at the six polymorphic positions $282,341,481,590,803$, and 857) to provide an inferred acetylation status that was compared to the "true" one predicted from the diplotype configuration at NAT2. The web-server was used in the standard way, by specifying the genotype at each polymorphic position using radio buttons and clicking the 'Submit' button. We retained for each subject the final prediction (i.e., the phenotype with the highest probability). The performance of NAT2PRED was measured by computing in each sample the classification error rate, which is the proportion of individuals whose acetylation status was incorrectly inferred by the web-server. The classification error rate of NAT2PRED was evaluated by considering either two (slow vs. others) or three (slow vs. intermediate vs. rapid) phenotypic classes for the acetylation status.

\section{Results and discussion}

The classification error rates of NAT2PRED in each sample for both classification issues (two or three acetylation phenotypes) are shown in Figure 1 (see Additional File 1 for the exact numbers). The performance of this tool in each of the five world regions investigated is summarized in
Table 1. NAT2PRED performed poorly in almost all subSaharan African samples investigated, except in the two Ethiopian samples where it correctly classified $100 \%$ of subjects into rapid, intermediate and slow acetylators. Such a poor performance is not surprising in view of the omission by the supervised learning classifier implemented in NAT2PRED of the functional SNP 191G $>$ A which, although monomorphic in most worldwide populations, occurs at an appreciable frequency in many subSaharan African populations (up to 23\%) and accounts for a sizeable portion of the slow acetylators encountered in these populations $[11,12]$. By contrast, NAT2PRED achieved a high classification accuracy in all the other world regions. In particular, individuals with a slow acetylation phenotype were correctly identified as slow acetylators with a sensitivity above 99\% for all populations outside sub-Saharan Africa. Conversely, NAT2PRED showed a poor ability to distinguish between intermediate and rapid acetylators, with a classification error rate reaching up to $10 \%$ in the non-African samples.

It is important to note that for most of the samples investigated, NAT2 haplotypes were not actually determined by a molecular resolution of mutation linkage patterns but were rather inferred through computational methods (see Additional File 1). As real NAT2 haplotypes may be quite different from those calculated by computational inference [7], it is of high relevance to evaluate the performance of NAT2PRED in only those samples where the real NAT2 haplotypes were determined. There are only six population samples where molecular techniques were used to reconstruct haplotypes and applied to the whole set of individuals. In the single African sample (Tswana from South Africa), NAT2PRED showed a poor classification accuracy with a classification error rate of $10 \%$ and $16 \%$ when considering two (slow vs. others) or three

Table I: Classification error rates of NAT2PRED in each geographic regiona.

\begin{tabular}{lcc}
\hline & \multicolumn{2}{c}{ Classification error rate (\%) } \\
\cline { 2 - 3 } & Two acetylation phenotypes ${ }^{\mathbf{b}}$ & Three acetylation phenotypes c \\
\hline Sub-Saharan Africa $(n=617)$ & $6.81 \%[0-14 \%]$ & $14.26 \%[0-30 \%]$ \\
Europe and North Africa $(n=4,391)$ & $0.32 \%[0-1 \%]$ & $0.98 \%[0-8 \%]$ \\
Central/South Asia $(n=757)$ & $0.53 \%[0-1 \%]$ & $3.57 \%[0-10 \%]$ \\
East Asia $(n=2,346)$ & $0.09 \%[0-0.2 \%]$ & $1.71 \%[0-8 \%]$ \\
America $(n=378)$ & $0 \%$ & $2.12 \%[0-3 \%]$ \\
\hline
\end{tabular}

Values are the classification error rates of NAT2PRED when all population samples within a geographic area were pooled into a single sample. The range of variation of the classification error rate across individual samples is indicated in square brackets.

aSee Figure I for the population composition of each geographic area.

bClassification error rate when two phenotypic classes are considered: slow vs. other acetylators (intermediate and rapid acetylators pooled together).

cClassification error rate when three phenotypic classes are considered: slow, intermediate and rapid acetylators. 


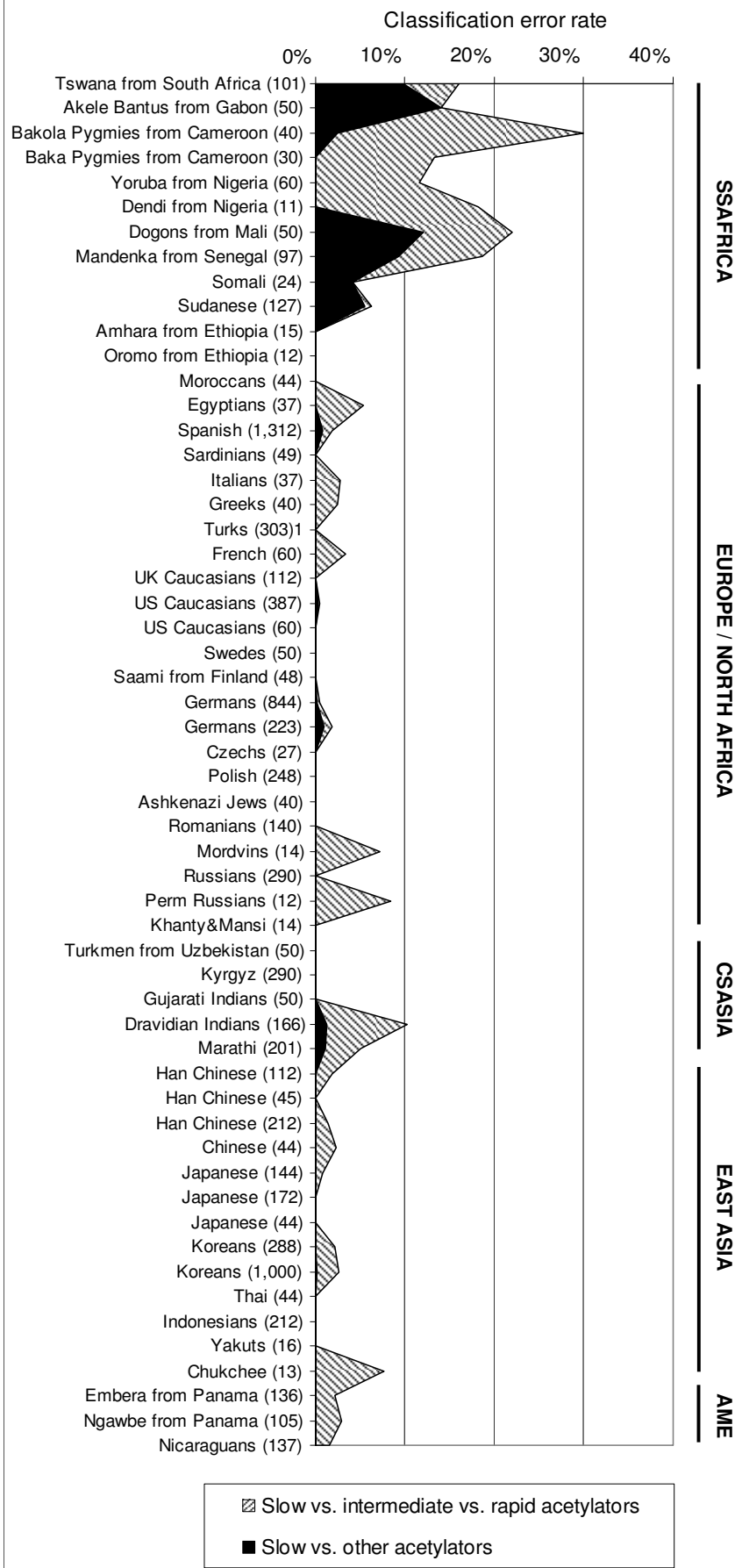

\section{Figure I}

Classification error rates of NAT2PRED in the $\mathbf{5 6}$ worldwide samples. The classification accuracy of NAT2PRED was evaluated using either three phenotypic classes (slow, intermediate and rapid acetylators; hatched area) or two phenotypic classes (slow and other acetylators; black area) for the acetylation status. Single populations are reported on the left side of the plot, with sample sizes (number of individuals) in brackets. Geographic areas are indicated on the right side, as follows: SSAFRICA, sub-Saharan Africa; EUROPE/NORTH AFRICA, Europe and North Africa; CSASIA, Central and South Asia; EAST ASIA, East Asia; AME, America. The assignment of populations to one of the five world regions was based on the origin of the population, in effect ignoring the past I,000 years of known human migration (e.g., people of European descent in the United States were assigned to Europe). 
(slow vs. intermediate vs. rapid) phenotypic classes for the acetylation status, respectively. In the five remaining nonAfrican samples, the ability of NAT2PRED to distinguish slow acetylators from the others was very high with an average classification error rate of only $0.5 \%$, ranging from $0 \%$ in UK Caucasians and Nicaraguans to $1.2 \%$ in Dravidian Indians. This means that in $99.5 \%$ of cases, NAT2PRED correctly identified slow acetylators in the same way the usual procedure did (that is, by reconstructing the real haplotypes through molecular techniques and by inferring the individual acetylation status from the haplotype combination at NAT2). On the other hand, a poorer performance was observed when intermediate acetylators were to be distinguished from rapid acetylators in these five samples (average error rate of $2.6 \%$, ranging from $0 \%$ to $10.2 \%$ ).

\section{Conclusion}

Our results show that NAT2PRED is an excellent tool to infer the individual acetylation status from NAT2 genotype data when the main interest is to distinguish slow acetylators from the others. Its classification error rate is indeed too high to ensure a reliable discrimination between intermediate and rapid acetylators. However, since the slow acetylator phenotype is the one which exhibits today the strongest association with susceptibility to bladder cancer and adverse drug reactions $[2,3]$, the web-server NAT2PRED should be of great use in clinical laboratories. This should facilitate the determination of the individual acetylation status in routine clinical practice and lead to better monitoring of risks associated with cancer and adverse drug reactions. On the other hand, NAT2PRED must be used with caution for patients whose population of origin contains a sizeable frequency of SNP $191 \mathrm{G}>\mathrm{A}$. This concerns populations of African origin but also several non-African populations, notably SouthEuropean and South-American populations, where this SNP has been identified, probably as a consequence of genetic admixture with Africans [13]. In particular, a frequency above $1 \%$ has been reported for the $191 \mathrm{~A}$ allele in Spanish (1.4\%) [7], in Coyaima from Colombia (3.5\%) [14], in Brazilians from Rio de Janeiro (4.7\%) [15], and in Brazilians from Goiàs (2.4\%) [15].

\section{Competing interests}

The authors declare that they have no competing interests.

\section{Authors' contributions}

AS: planned the analysis, collected and analyzed the data, drafted the manuscript. PD and MV: participated in the interpretation of data and critically revised the manuscript. All authors have read and approved the content of the manuscript.

\section{Additional material}

\section{Additional file 1}

Table S1. Classification error rates of NAT2PRED in the 56 worldwide samples collected from the literature.

Click here for file

[http://www.biomedcentral.com/content/supplementary/14712350-10-148-S1.DOC]

\section{References}

I. Meisel P: Arylamine $\mathbf{N}$-acetyltransferases and drug response. Pharmacogenomics 2002, 3(3):349-366.

2. Ladero JM: Influence of polymorphic $\mathbf{N}$-acetyltransferases on non-malignant spontaneous disorders and on response to drugs. Curr Drug Metab 2008, 9(6):532-537.

3. Agúndez JA: Polymorphisms of human $\mathbf{N}$-acetyltransferases and cancer risk. Curr Drug Metab 2008, 9(6):520-53I.

4. García-Closas M, Malats N, Silverman D, Dosemeci M, Kogevinas M, Hein DW, Tardón A, Serra C, Carrato A, García-Closas R, Lloreta J, Castaño-Vinyals G, Yeager M, Welch R, Chanock S, Chatterjee N, Wacholder S, Samanic C, Torà M, Fernández F, Real FX, Rothman N: NAT2 slow acetylation, GSTMI null genotype, and risk of bladder cancer: results from the Spanish Bladder Cancer Study and meta-analyses. Lancet 2005, 366(9486):649-659.

5. Hein DW: $\mathbf{N}$-acetyltransferase 2 genetic polymorphism: effects of carcinogen and haplotype on urinary bladder cancer risk. Oncogene 2006, 25(I I):1649-1658.

6. Batra J, Ghosh B: $\mathbf{N}$-acetyltransferases as markers for asthma and allergic/atopic disorders. Curr Drug Metab 2008, 9(6):546-553.

7. Agúndez JA, Golka K, Martínez C, Selinski S, Blaszkewicz M, GarcíaMartín E: Unraveling ambiguous NAT2 genotyping data. Clin Chem 2008, 54(8): 1390-1394.

8. Sabbagh A, Darlu P: Inferring haplotypes at the NAT2 locus: the computational approach. BMC Genet 2005, 6(I):30.

9. Golka K, Blaszkewicz M, Samimi M, Bolt HM, Selinski S: Reconstruction of $\mathbf{N}$-acetyltransferase 2 haplotypes using PHASE. Arch Toxicol 2008, 82(4):265-270.

10. Kuznetsov IB, McDuffie M, Moslehi R: A web-server for inferring the human $\mathbf{N}$-acetyltransferase-2 (NAT2) enzymatic phenotype from NAT2 genotype. Bioinformatics 2009, 25(9): I I85- I 186 .

II. Sabbagh A, Langaney A, Darlu P, Gérard N, Krishnamoorthy R, Poloni ES: Worldwide distribution of NAT2 diversity: implications for NAT2 evolutionary history. BMC Genet 2008, 9:21.

12. Patin E, Harmant C, Kidd KK, Kidd J, Froment A, Mehdi SQ, Sica L, Heyer E, Quintana-Murci L: Sub-Saharan African coding sequence variation and haplotype diversity at the NAT2 gene. Hum Mutat 2006, 27(7):720.

13. García-Martín E: Interethnic and intraethnic variability of NAT2 single nucleotide polymorphisms. Curr Drug Metab 2008, 9(6):487-497.

14. Fuselli S, Gilman RH, Chanock SJ, Bonatto SL, De Stefano G, Evans CA, Labuda D, Luiselli D, Salzano FM, Soto G, Vallejo G, Sajantila A, Pettener D, Tarazona-Santos E: Analysis of nucleotide diversity of NAT2 coding region reveals homogeneity across Native American populations and high intra-population diversity. Pharmacogenomics J 2007, 7(2): 144-152.

15. Teixeira RL, Miranda AB, Pacheco AG, Lopes MQ, Fonseca-Costa I, Rabahi MF, Melo HM, Kritski AL, Mello FC, Suffys PN, Santos AR: Genetic profile of the arylamine $\mathbf{N}$-acetyltransferase 2 coding gene among individuals from two different regions of Brazil. Mutat Res 2007, 624(I-2):3 I-40.

\section{Pre-publication history}

The pre-publication history for this paper can be accessed here: 
http://www.biomedcentral.com/1471-2350/10/148/pre

pub

Publish with Biomed Central and every scientist can read your work free of charge

"BioMed Central will be the most significant development for disseminating the results of biomedical research in our lifetime. " Sir Paul Nurse, Cancer Research UK

Your research papers will be:

- available free of charge to the entire biomedical community

- peer reviewed and published immediately upon acceptance

- cited in PubMed and archived on PubMed Central

- yours - you keep the copyright 